\title{
ERRATUM TO: "ACTION OF A LOCAL TIME-PERIODIC LOAD ON AN ICE SHEET WITH A CRACK"
}

\section{[Journal of Applied Mechanics and Technical Physics}

\section{8 (6), 1069-1082 (2017)]}

\section{A. Tkacheva}

DOI: $10.1134 /$ S0021894418020232

In the original publication, the second formula on page 1077 was misspelled.

It should read:

$$
\begin{gathered}
\sum_{j=-2}^{\infty} \frac{\left(\alpha_{j}^{2}+\nu s^{2}\right)\left(a_{1} \alpha_{j}+a_{3} \alpha_{j}^{3}\right)}{K_{2}^{\prime}\left(\alpha_{j}, s\right)\left(\beta_{2} \mu_{j}^{4}+1-\delta_{2}\right)}=\frac{1}{2 \pi i} \int_{-\infty}^{\infty} \frac{\left(\alpha^{2}+\nu s^{2}\right)\left(a_{1} \alpha+a_{3} \alpha^{3}\right) d \alpha}{K_{2}(\alpha, s)\left(\beta_{2}\left(\alpha^{2}+s^{2}\right)^{2}+1-\delta_{2}\right)} \\
-\sum_{k=1}^{2} \frac{\left(\eta_{k}^{2}+\nu s^{2}\right)\left(a_{1} \eta_{k}+a_{3} \eta_{k}^{3}\right)}{K_{2}\left(\eta_{k}\right) 4 \beta_{2} \eta_{k}\left(\eta_{k}^{2}+s^{2}\right)}=-\sum_{j=-2}^{\infty} \frac{\left(\alpha_{j}^{2}+\nu s^{2}\right)\left(-a_{1} \alpha_{j}-a_{3} \alpha_{j}^{3}\right)}{K_{2}^{\prime}\left(-\alpha_{j}, s\right)\left(\beta_{2} \mu_{j}^{4}+1-\delta_{2}\right)}+\frac{1}{4 \beta_{2} \lambda^{2}} \sum_{k=1}^{4} \frac{\left(\eta_{k}^{2}+\nu s^{2}\right)\left(a_{1} \eta_{k}+a_{3} \eta_{k}^{3}\right)}{\eta_{k}\left(\eta_{k}^{2}+s^{2}\right)} \\
\eta_{k}= \pm\left( \pm i\left(\frac{1-\delta_{2}}{\beta_{2}}\right)^{1 / 2}-s^{2}\right)^{1 / 2} .
\end{gathered}
$$

The online version of the original article can be found under DOI: 10.1134/S002189441706013X.

Lavrent'ev Institute of Hydrodynamics, Siberian Branch, Russian Academy of Sciences, Novosibirsk, 630090 Russia; tkacheva@hydro.nsc.ru. Translated from Prikladnaya Mekhanika i Tekhnicheskaya Fizika, Vol. 58, No. 6, pp. 133-148, November-December, 2017. Original article submitted August 3, 2016; revision submitted September 5, 2016. 